\title{
Flux variability in the FSRQ 1510-089. A radio-gamma perspective
}

\author{
M. Orienti ${ }^{* \dagger}$, G. Giovannini, D. Dallacasa \\ Astronomy Department, Bologna University, Italy \\ E-mail: orienti@ira.inaf.it
}

M. Giroletti, T. Venturi

INAF-IRA Bologna, Italy

\section{F. D'Ammando, S. Vercellone}

INAF-IASF Palermo, Italy

\section{Tavani}

INAF-IASF Roma, Italy

The knowledge of the physical conditions occurring in the relativistic jet of radio-loud active galactic nuclei (AGNs) is important to understand the mechanisms at the basis of their multiband emission. From parsec-scale radio observations of blazar objects it has been suggested a connection between the ejection of new jet features and strong $\gamma$-ray flares. We present results from multi-epoch Very Long Baseline Interferometer (VLBI) and Space-VLBI observations of the Flat Spectrum Radio Quasar (FSRQ) PKS 1510-089. The comparison of the radio structure observed at different epochs shows the presence of jet features moving with highly superluminal apparent velocity. Radio flux density variability and changes in the source structure and in the polarization properties are then compared with the information on the $\gamma$-ray emission in order to find a possible connection between radio and $\gamma$-ray emission.

10th European VLBI Network Symposium and EVN Users Meeting: VLBI and the new generation of radio arrays

September 20-24, 2010

Manchester Uk

\section{* Speaker.}

$\dagger$ This research has made use of the data from the MOJAVE database that is maintained by the MOJAVE team (Lister et al. 2009, AJ, 137, 3718) 


\section{Introduction}

Only a small fraction of the AGN population display a powerful radio emission. This phenomenon is associated with relativistic particles produced in the central region of the AGN and channelled through the jets towards the outermost regions. In addition to the synchrotron radiation detected in the radio band, the relativistic particles may be also responsible for the high-energy emission by means of scattering of low-energy photons, suggesting in this way a connection between radio and $\gamma$-ray emission. The fact that all the AGN detected by EGRET were powerful radio-loud objects strongly support a relation between radio and $\gamma$-ray emission. However, such a relation has not been proved unambiguously. The class of AGN dominating the $\gamma$-ray sky is the blazar population. These objects are characterized by the presence of a compact radio core, superluminal jets, and extreme flux density and polarization variability. Their properties are interpreted as the result of severe beaming effects caused by the orientation of the jet axis at very small angles with the line of sight. Among the blazar population, the flat spectrum radio quasar PKS 1510-089 $(z=0.361)$ is the ideal target to locate the region responsible for the emission. Enhanced $\gamma$-ray activities have been detected several times by both AGILE and Fermi satellites [1, 2, 9]. In the radio band the source is characterized by highly superluminal jet knots with apparent velocity of about 20c [4]. Thanks to the very small angle of a few degrees formed by the jet with the line of sight, PKS 1510-089 represents the good candidate to investigate the possible connection between radio and $\gamma$-ray emission.

\section{The source morphology}

To study the parsec-scale morphology, PKS 1510-089 was target of multi-epoch Space-VLBI observations at $5 \mathrm{GHz}$ and VLBA observations at $8.4 \mathrm{GHz}$. The excellent spatial resolution ( $1.8^{\prime \prime} \times 0.9^{\prime \prime}$ at $5 \mathrm{GHz}$ and $\sim 2.3^{\prime \prime} \times 1.0^{\prime \prime}$ at $8.4 \mathrm{GHz}$ ) allowed us to accurately describe the source structure and to follow the ejected knots during their motion. Additional multi-epoch VLBA 15$\mathrm{GHz}$ data from the MOJAVE programme [6] were analysed in order to complement the morphological information with data at a higher frequency and to study on a longer time baseline the flux density and polarization variability of the core component. More details on the observations and the data analysis can be found in [8].

Dual-epoch Space-VLBI observations at $5 \mathrm{GHz}$ (Fig. 1a) show that the parsec scale radio emission of PKS 1510-089 has a core-jet structure, as pointed out by previous observations $[4,5]$. The radio emission is dominated by the core component $\mathrm{C}$ from which the jet emerges forming an angle of $-28^{\circ}$. The jet emission is not straight, but it slightly bends at about 10 parsec from the core. Deep multi-epoch polarimetric VLBI observations at $8.4 \mathrm{GHz}$ (Fig. 1b) point out the presence of a lowsurface brightness tail visible up to $125 \mathrm{pc}$. The emission from the jet is mainly from the first $25 \mathrm{pc}$, where various knots ejected from the core at different times can be identified and followed throughout several observing epochs. For example, by comparing 15-GHz VLBA observations from the multi-epoch MOJAVE programme it is possible to identify different jet knots and monitor their evolution (see Section 3). At $8.4 \mathrm{GHz}$ both the core and the jet are polarized in all the observing epochs. 

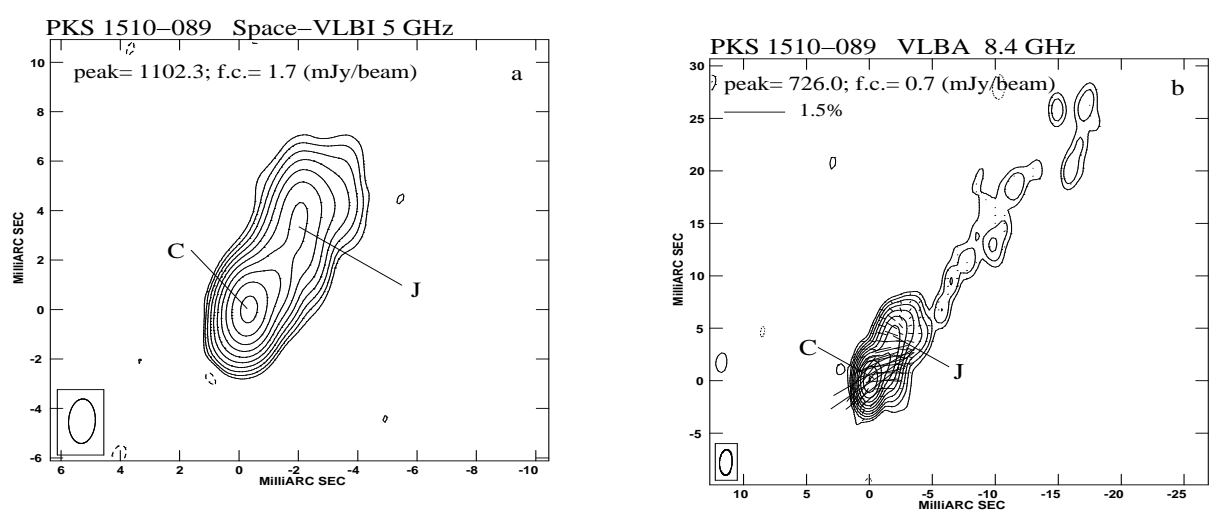

Figure 1: 5-GHz Space-VLBI (left) and 8.4-GHz VLBA (right) images of PKS 1510-089. The first contour (f.c.) level corresponds to three time the off-source noise level. Contours increase by a factor 2. Adapted from [8].

\section{Proper motion}

Multi-epoch analysis of the pc-scale morphology shows an evolution of the source structure: non-stationary jet components are ejected at different times and their changes can be followed by comparing observations carried out after short time intervals. From the comparison of the threeepoch data at $8.4 \mathrm{GHz}$ obtained between 1999 and 2001, we found that component $\mathrm{J}$ is moving away from the central component $C$ with a highly superluminal apparent separation velocity $\beta=15.9 \pm 0.3$ [8]. To investigate the possible connection between ejection of new jet components and $\gamma$-ray flares, we analysed multi-epoch 15-GHz VLBA data spanning a time interval from 2007 and 2010, i.e. in the AGILE and Fermi era. During this time interval we could follow the evolution of three knots with apparent velocity between $16 c$ and $19.5 c$. In two cases regression extrapolation suggests that the ejection of the knot occurred very close in time with a strong $\gamma$-ray flare, as also found by [7], suggesting a connection between the radio and $\gamma$-ray emission.

\section{Flux density variability and polarization}

The analysis of the light-curve of PKS 1510-089 shows strong flux density variability where periods in which the source is in a low state are alternated with flares [11]. The pc-scale resolution of the MOJAVE data allows us to separate the contribution of the core from that of the jet structure. The comparison between the radio variability and polarimetric information of the core and the episodes of enhanced $\gamma$-ray luminosity does not provide a clear picture. Indeed, the high $\gamma$-ray activity states occurred in September 2007 [9] and September 2008 [10] seem to follow a radio flare, while the enhanced activity observed in March 2009 [3] seems to coincide with a low state in the radio band (Fig. 2a). If we consider the polarization properties, it seems that the polarization percentage does not correlate with the $\gamma$-ray and total radio flux density variability (Fig. 2b). On the other hand, the polarization angle (Fig. 2c) shows an abrupt change of about $75^{\circ}$ close in time with the strong increment in both radio and $\gamma$-ray emission [8]. The fact that such a rotation of the polarization angle occurs close in time with the ejection of a superluminal component may indicate 


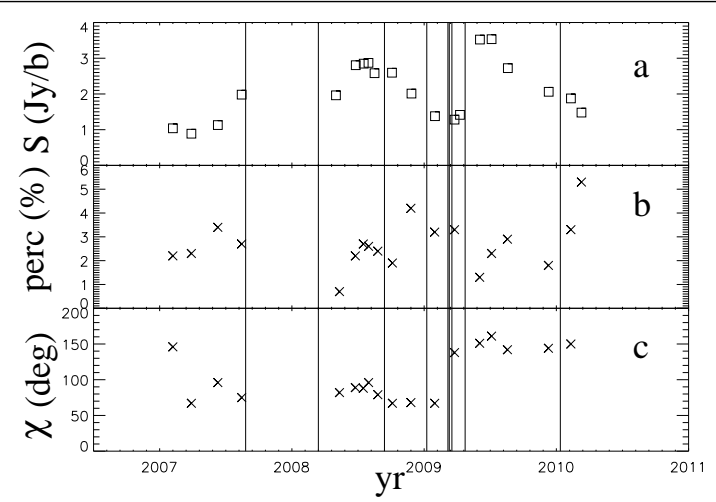

Figure 2: Multi-epoch 15-GHz flux density $(a)$, polarization percentage $(b)$, and polarization angle $(c)$ of the core component of PKS 1510-089. Adapted from [8].

that the new component is highly polarized with an orientation of the electric vectors that is very different from that of the core.

\section{Summary}

We have presented multi-epoch high spatial resolution VLBI and Space-VLBI observations of the core-jet FSRQ PKS 1510-089. The comparison of the pc-scale radio structure observed at different epochs shows the presence of non-stationary jet components moving at highly superluminal velocity. For two of these knots, the time of zero separation from the core occurs close to an episode of enhanced $\gamma$-ray activity. After these episodes, changes in the radio flux density and in polarization properties have been observed, and they may be due to the ejected component, spatially unresolved from the core region, possessing a different orientation of the magnetic field with respect to the core.

\section{References}

[1] Abdo, A.A., Ackermann, M., Agudo, I., et al. 2010, ApJ, 721, 1425

[2] D’Ammando, F., Pucella, G., C.M. Raiteri, et al. 2009, A\&A, 508, 181

[3] D’Ammando, F., Raitieri, C.M., Villata, M., et al. 2010, A\&A, submitted

[4] Homan, D.C., Wardle, J.F.C., Cheung, C.C., Roberts, D.H., Attridge, J.M. 2002, ApJ, 580, 742

[5] Jorstad, S.G., Marsher, A.P., Lister, M.L., et al. 2005, AJ, 130, 1418

[6] Lister, M.L., Aller, H.D., Aller, M.F., et al. 2009, AJ, 137, 3718

[7] Marscher, A.P., Jorstad, S.G., Larionov, V.M., et al. 2010, ApJL, 710, 126

[8] Orienti, M., Venturi, T., Dallacasa, D., et al. 2010, A\&A, submitted

[9] Pucella, G., Vittorini, V., D'Ammando, F., et al. 2008, A\&A, 491, 21

[10] Tramacere, A. 2008, ATel, 1743

[11] Venturi, T., Dallacasa, D., Orfei, A., et al. 2001, A\&A, 379, 755 